\title{
Implementation of a finite element model for stress analysis of gear drives based on multi-point constraints
}

\author{
Ignacio Gonzalez-Perez ${ }^{\mathrm{a}, *}$, Alfonso Fuentes-Aznar ${ }^{\mathrm{b}}$ \\ ${ }^{a}$ Polytechnic University of Cartagena, Department of Mechanical Engineering, Cartagena, Murcia, SPAIN \\ ${ }^{b}$ Rochester Institute of Technology, Department of Mechanical Engineering, Rochester, NY, USA
}

\begin{abstract}
Gear researchers are always trying to find a trade-off solution between obtaining accurate results from gear stress analyses and low computational costs. Both factors, accuracy on the one hand, and a low computational cost on the other hand, usually go in opposite directions. In this paper, a finite element model for stress analysis of gear drives is proposed with the ultimate goal of obtaining accurate results regarding contact and bending stresses with lower computational cost than other finite element models presented in the literature. The proposed finite element model allows the whole cycle of meshing to be analyzed and is based on the application of multi-point constraints for mesh refinement and the application of elements with a reduced number of integration points. Node coordinates are computationally and automatically determined by application of the gear theory. Several numerical examples are presented.
\end{abstract}

Keywords: Gear Stress Analysis, Finite Element Method.

\section{Introduction}

Stress analysis is an important aspect in the design and the analysis of gear drives. The solution of the stress analysis should provide the actual size of the contact pattern due to the transmitted load, the contact stresses on the contacting surfaces and underneath the contacting surfaces, the bending stresses at the fillet of the gear teeth, and the surface deformation for the calculation of loaded transmission errors.

Many approaches for gear stress analysis have been applied so far. Many of them are based on the application of the finite element method in which this paper is focused on. First applications of the finite element method in gears were directed towards the determination of root bending stresses as in [1] for a two dimension analysis and in [2] for a three dimension analysis. Later, finite element analyses covered as well the contact problem as in [3] for a two dimension analysis and in [4] for a three dimension analysis. The analysis of the stress evolution along a whole cycle of meshing and the automatic determination of the node coordinates were steps forward in the application of the finite element method to gears [5]. Some improvements of the finite element model to consider torsional deformation of the gears and the effect of shaft deflections came later $[6,7]$.

\footnotetext{
* Corresponding author

Email address: ignacio.gonzalez@upct.es (Ignacio Gonzalez-Perez)
} 
Some other approaches for stress analysis of gears are based on numerical methods that combine analytical solutions with finite element analysis. Those methods are in general more computational effective than those that are based exclusively on the application of the finite element method [8]. Other numerical methods with reduced computational cost are based on the application of shell theory to gears [9]. Although finite element analysis can be very accurate, in general its main drawback is the computational cost derived from the amount of finite elements that are required around the contact area and along the fillet profile. First examples of reduction of the number of finite elements in the fillet region of the gear teeth by means of transition elements can be found in [10] for a three dimension problem. Here, transition elements in the contact region for a two dimension problem were applied as well. Later, a reduction of the number of elements based on the application of surface-based tie constraints was applied in [11]. The high computational cost of many finite element models is due mainly to the symmetry of the mesh in both, driving and coast sides, as the finite element model proposed in [5] and shown in Fig. 1 for the case of a spur gear drive.

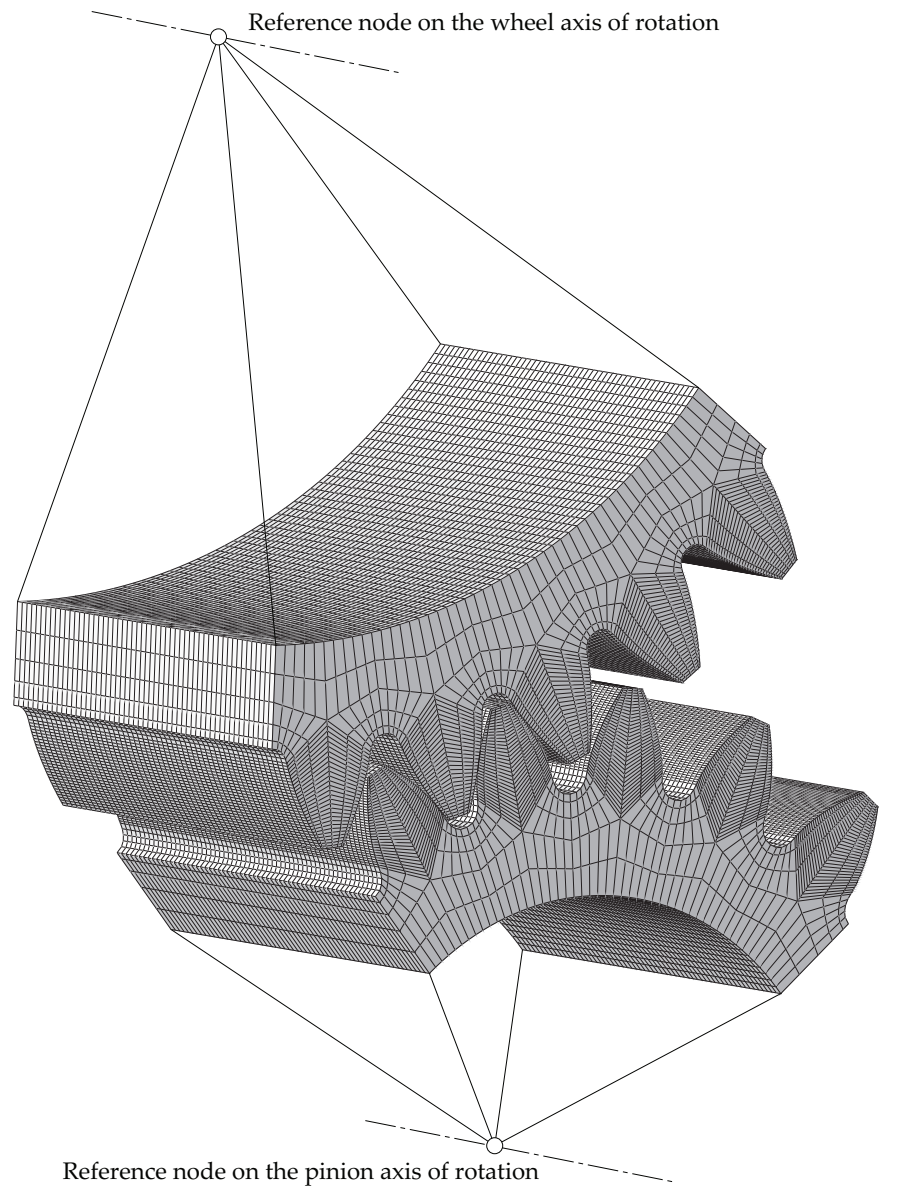

Figure 1: Example of a finite element model that requires a high computational cost.

In the characterization of gear materials, several magnitudes regarding strength are managed. Regarding the contact area, a fatigue strength for Hertzian pressure and a tensile strength can be considered. Maximum contact pressure and effective Tresca or Von Mises stresses should be taken 
into account for comparison with those magnitudes previously mentioned, respectively. Regarding the bending area, a fatigue strength for tooth root stress is usually considered. For such a value, the maximum principal stress should be taken into account for comparison.

The main goals of this research are summarized as follows and are based on the application of a finite element model that allows to:

(i) Get accurate results of the maximum value of contact pressure on the contacting surfaces.

(ii) Get accurate results of the maximum value of Tresca or Von Mises effective stress underneath the contacting surfaces.

(iii) Get accurate results of the maximum principal stress at the fillet of gear teeth.

(iv) Analyze a whole cycle of meshing and obtain results for several contact positions and not just for one contact position.

(v) Reduce the computational cost respect to existing finite element models.

The proposed finite element model will be validated by means of comparison of the obtained results with those provided through the application of Hertz theory when it can be applied, or by means of the observation of convergency of the results through mesh refinement. Several numerical examples are presented. The proposed ideas are valid for most types of gear geometries, although worm geometries would require some adjustments. Accuracy of the results and computational cost are compared to previous finite element models as the one shown in Fig. 1.

\section{Finite element model definition}

The definition of the proposed finite element model is based upon the following steps:

- Step 1. Point coordinates on the gear tooth surfaces, either for profile or fillet regions, are determined analytically considering the gear type, its generating tool and the cutting method through the application of the gear theory [12]. These coordinates and the coordinates of some points defined on the gear rim are considered to design the gear tooth volume. Fig. 2(a) shows the points that are considered to form the designed volume of one gear tooth.

- Step 2. The designed gear tooth volume is divided considering six intermediate auxiliary surfaces as it is illustrated in Fig. 2(b). A total of eight layers of elements will therefore be considered in the mesh of the designed gear tooth volume. Layers can be managed independently from each other as it will be shown later.

- Step 3. Nodes on the gear tooth surfaces, on the gear rim, and inside the designed volume, are automatically determined (see Fig. 2(c)) from the previous defined point coordinates, following the arrangement of layers shown in Fig. 2(b) and according to a strategy for mesh refinement based on multi-point constraints that will be exposed later. A reduced number of nodes are illustrated here for the purpose of clarity.

- Step 4. Linear elements are formed from the previously defined nodes and following the connectivity that is shown in Fig. 2(d). 

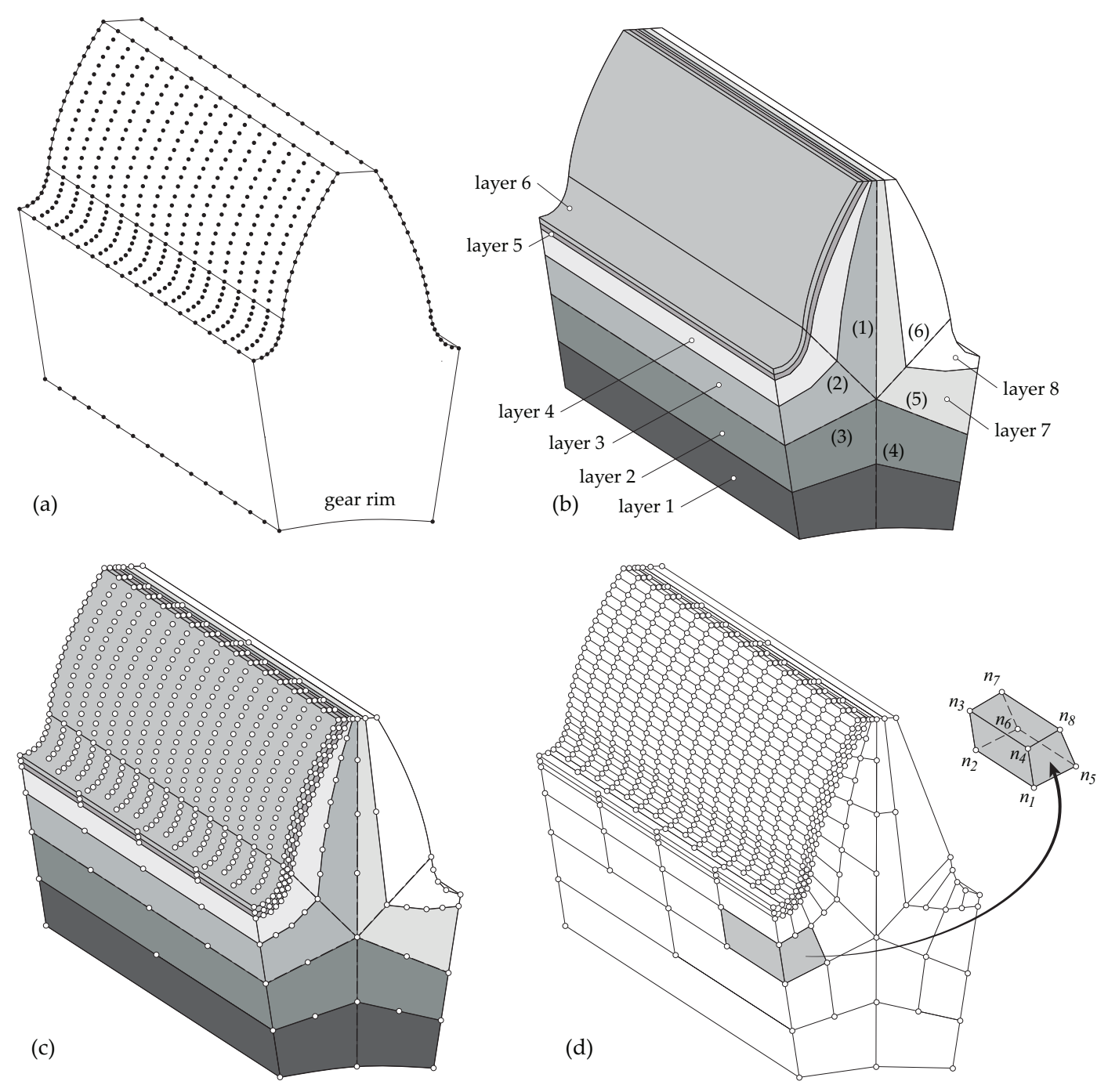

Figure 2: For finite element model definition: (a) points, (b) imaginary layers and intermediate surfaces, (c) nodes, and $(\mathrm{d})$ finite elements.

- Step 5. Nodes defined on both sides and the bottom part of the gear rim constitute a rigid surface that is rigidly connected to a reference node defined in the gear axis as it is illustrated in Fig. 3. A torque is applied at such a reference node in the pinion model whereas the reference node of the wheel model is held at rest.

- Step 6. Some contact positions are analyzed through consideration of several load steps. At each load step, the reference node defined in the wheel axis of rotation is rotated an angle provided by the tooth contact analysis of the gear drive and then held at rest, while the same torque is kept applied to the pinion reference node during the whole analysis.

\subsection{Multi-point constraints}

A strategy for mesh refinement based on the application of multi-point constraints has been applied across the whole model. Such a strategy allows either refinement from two elements to one 


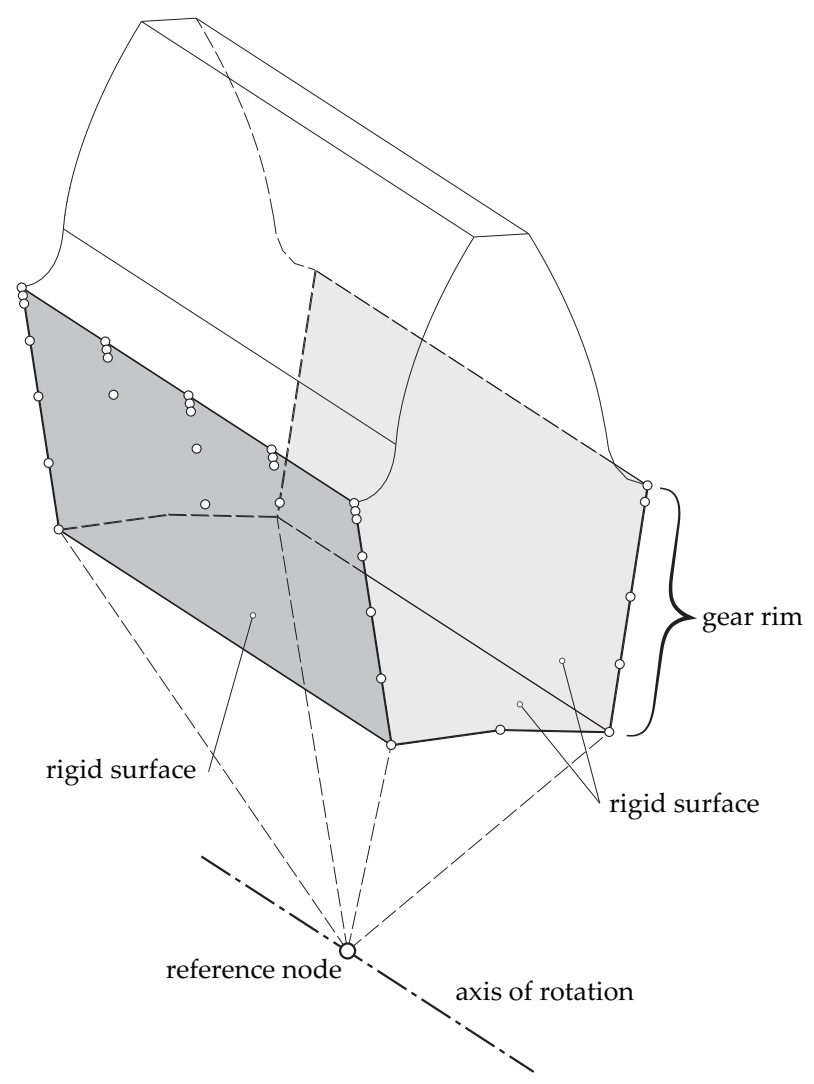

Figure 3: For definition of the rigid surface.

element or refinement from four elements to one element. Figure 4 shows the nodes involved in the connection between some elements that belong to layer 1 and layer 2 (see Fig. 2). In this case, a refinement from four elements in layer 2 to one element in layer 1 is presented.

Mesh transitions require application of constraints at the degrees of freedom of some nodes, called dependent nodes, as linear functions of the degrees of freedom of some other nodes, called independent nodes, in order to assure the continuity in the field of displacements. Figure 4 shows nodes $n_{2}, n_{4}, n_{5}, n_{6}$, and $n_{8}$, as dependent nodes, and nodes $n_{1}, n_{3}, n_{7}$, and $n_{9}$, as independent nodes. A degree of freedom dof of a dependent node can be defined as follows.

$$
\begin{aligned}
\operatorname{dof}_{n_{2}} & =f_{2}\left(\operatorname{dof}_{n_{1}}, \operatorname{dof}_{n_{3}}\right) \\
\operatorname{dof}_{n_{4}} & =f_{4}\left(\operatorname{dof}_{n_{1}}, \operatorname{dof}_{n_{7}}\right) \\
\operatorname{dof}_{n_{5}} & =f_{5}\left(\operatorname{dof}_{n_{1}}, \operatorname{dof}_{n_{3}}, \operatorname{dof}_{n_{7}}, \operatorname{dof}_{n_{9}}\right) \\
\operatorname{dof}_{n_{6}} & =f_{6}\left(\operatorname{dof}_{n_{3}}, \operatorname{dof}_{n_{9}}\right) \\
\operatorname{dof}_{n_{8}} & =f_{8}\left(\operatorname{dof}_{n_{7}}, \operatorname{dof}_{n_{9}}\right)
\end{aligned}
$$

Here, $f_{2}, f_{4}, f_{5}, f_{6}$ and $f_{8}$ are linear interpolation functions of the elements and are given by a general purpose computer program for stress analysis based on the finite element method [13]. A similar procedure is applied across the whole model. Those nodes that belong to either both sides of the rim or the bottom part of the rim and are considered dependent, are not used to define the 


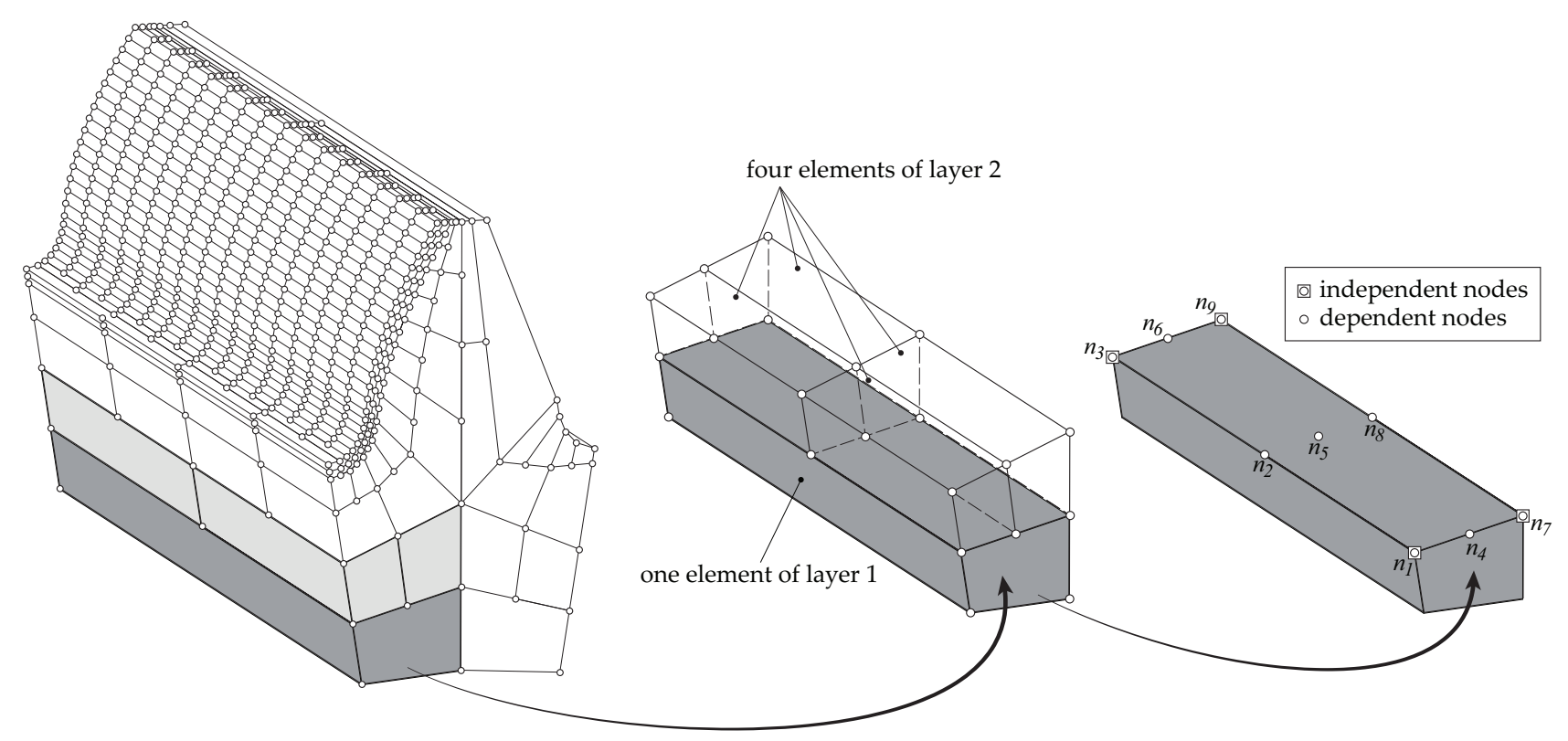

Figure 4: For illustration of multi-point constraints.

rigid surface shown in Fig. 3. Only independent nodes can be part of the rigid surface.

\subsection{Types of element formulations}

The finite element model is defined through linear elements of eight nodes. This type of element shows a better behavior in the determination of contact pressures respect to quadratic elements [13]. The arrangement of nodes and elements through eight layers (see Fig. 2) allows an independent selection of elements with different formulations for each layer. In this way, whereas layers 5 and 6 , which are closer to the areas of interest in the gear stress analysis, can be defined by means of linear elements with the complete number of integration points (a total of eight integration points for each element), the other layers can be defined by means of linear elements with a reduced number of integrations points (one integration point for each element). Whereas elements with the complete number of integration points allow a better accuracy of the results, the elements with a reduced number of integration points allow the computational cost to be decreased since stresses are evaluated at just one point. The drawback of reduced-integration elements is that they may behave more stiffly. This circumstance will be discussed in Section 3. Figure 5 shows a possible strategy for the arrangement of element formulations in the finite element model.

\subsection{Arrangement for mesh refinement and connection of several teeth}

The mesh of the model is arranged through a strategy for refinement either across the transverse sections of the gear teeth or along the axis of the gear. Figure 6 shows the transverse sections of two gear teeth in the finite element model for illustration of this arrangement. A number of elements in the active profile $n_{p}$ and a number of elements in the fillet $n_{f}$ are defined. These numbers of elements appear on layers 5 and 6 (see Fig. 2). As the mesh is spread across the transverse sections of the gear teeth, the number of elements are reduced by considering a multiplication factor of $1 / 2$ from layer to layer. Simultaneously, if a number of elements in longitudinal (along gear axis) direction $n_{l}$ is defined for layers 5 and 6 , the number of elements in longitudinal direction 


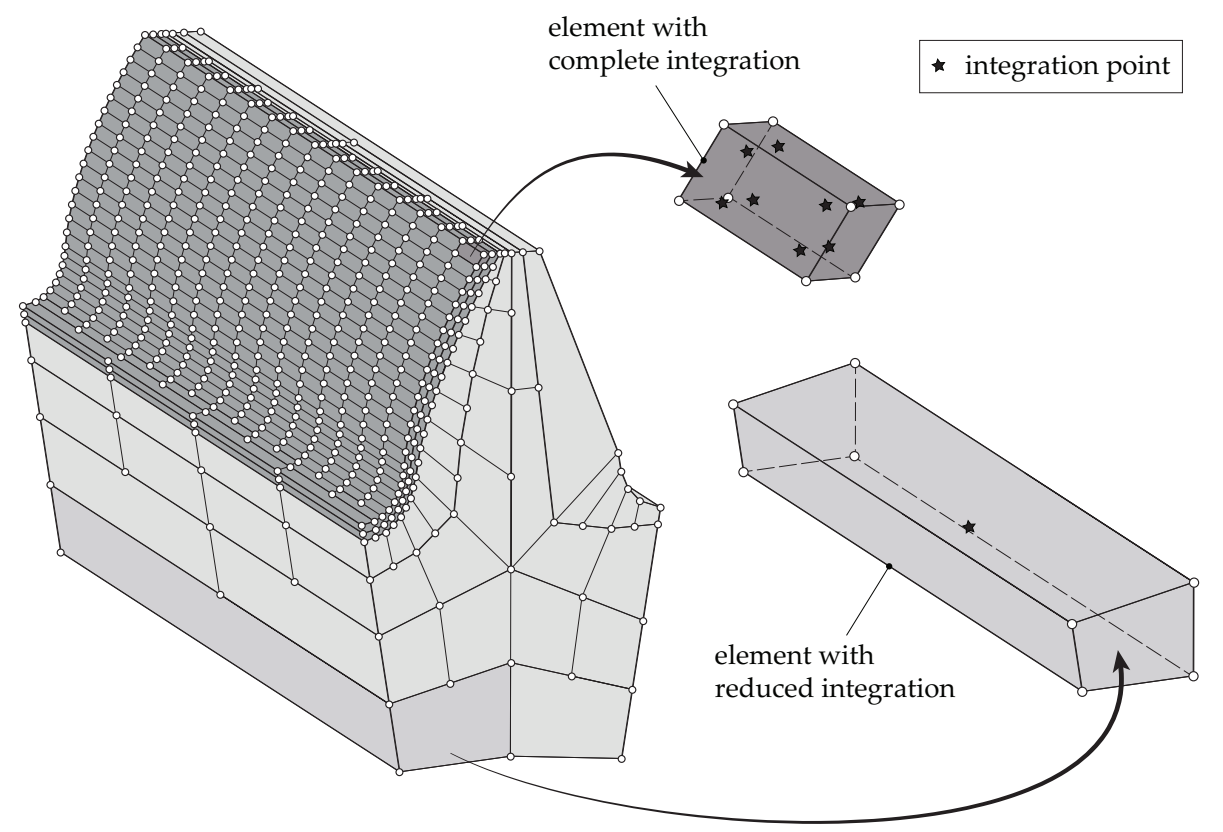

Figure 5: Arrangement for different formulations of the linear elements.

is reduced considering also a multiplication factor of $1 / 2$ from layer to layer. Since four reductions of elements are applied across the transverse section, the variables $n_{p}$ and $n_{l}$, that can be defined independently, are given as follows

$$
\begin{aligned}
n_{p} & =\left\{1 \cdot 2^{4}, 2 \cdot 2^{4}, 3 \cdot 2^{4}, 4 \cdot 2^{4}, 5 \cdot 2^{4}, \ldots\right\}=\{16,32,48,64,80, \ldots\} \\
n_{l} & =\left\{1 \cdot 2^{4}, 2 \cdot 2^{4}, 3 \cdot 2^{4}, 4 \cdot 2^{4}, 5 \cdot 2^{4}, \ldots\right\}=\{16,32,48,64,80, \ldots\}
\end{aligned}
$$

whereas for $n_{f}$ two possibilities have been considered: $n_{f}=\{8,16\}$.

Regarding the connection between the teeth, layer 8 of a given tooth is connected with layers 5 and 6 of the following tooth. A transition in the number of elements in longitudinal direction is required either on the fillet of layer 8 of the given tooth or the fillet of layers 5 and 6 of the following tooth to make the connection possible. Layer 7 of the given tooth is also connected with layers 3 and 4 of the following tooth. Layers 1 and 2 of adjacent teeth are also connected. Distances $a, b$, $c$, and $d$ have to be defined with the same values at both sides of the gear teeth for the connection of several teeth. Some nodes on the left side of the rim of the following tooth needs to be removed since they are already present in the given tooth. Rigid surface is also adjusted when several teeth are included into the model.

\subsection{Different models for both rotations}

Whereas the model shown in Fig. 1 allows for the analysis of stresses considering both rotations, counterclockwise and clockwise, and can be useful to detect contacts on the coast side due to a loss of backlash, the model proposed in this paper can only be applied for one direction of rotation. This means that a different finite element model is required for the analysis of stresses when the pinion rotates counterclockwise or when the pinion rotates clockwise. Figure 7 shows two models of a right-hand helical pinion for both rotation directions. 


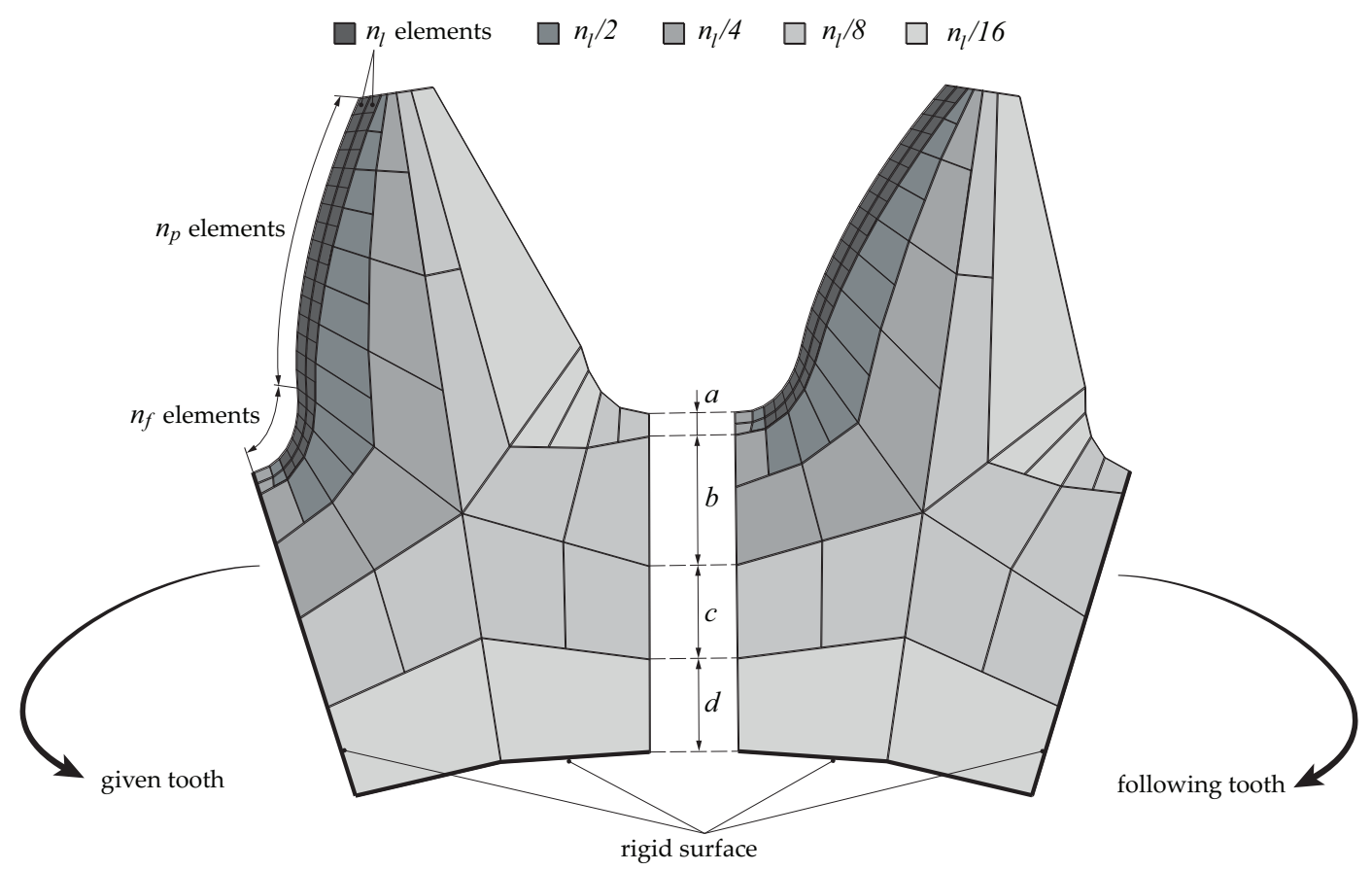

Figure 6: Arrangement for elements across the gear tooth sections and along the gear axis.

\section{Numerical examples}

The goals of this section are as follows:

(1) To prove the correctness of the results yielded by the proposed finite element model in terms of maximum contact pressure, maximum effective Tresca or Von Mises stress underneath the contacting surfaces, and maximum principal stress on the fillet area, all along the whole cycle of meshing.

(2) Comparison of the computational cost between the proposed model and the previous model shown in Fig. 1.

(3) Investigate the effects of multi-point constraints and reduced-integration elements in the accuracy of the results and computational cost.

(4) Investigate the effects of the thickness of layers 5 and 6 (see Fig. 2) in the results.

The contents of this section are:

(i) Validation of the proposed model with Hertz theory.

(ii) Comparison of the proposed model and the previous model.

(iii) Validation of the proposed model regarding bending stresses. 


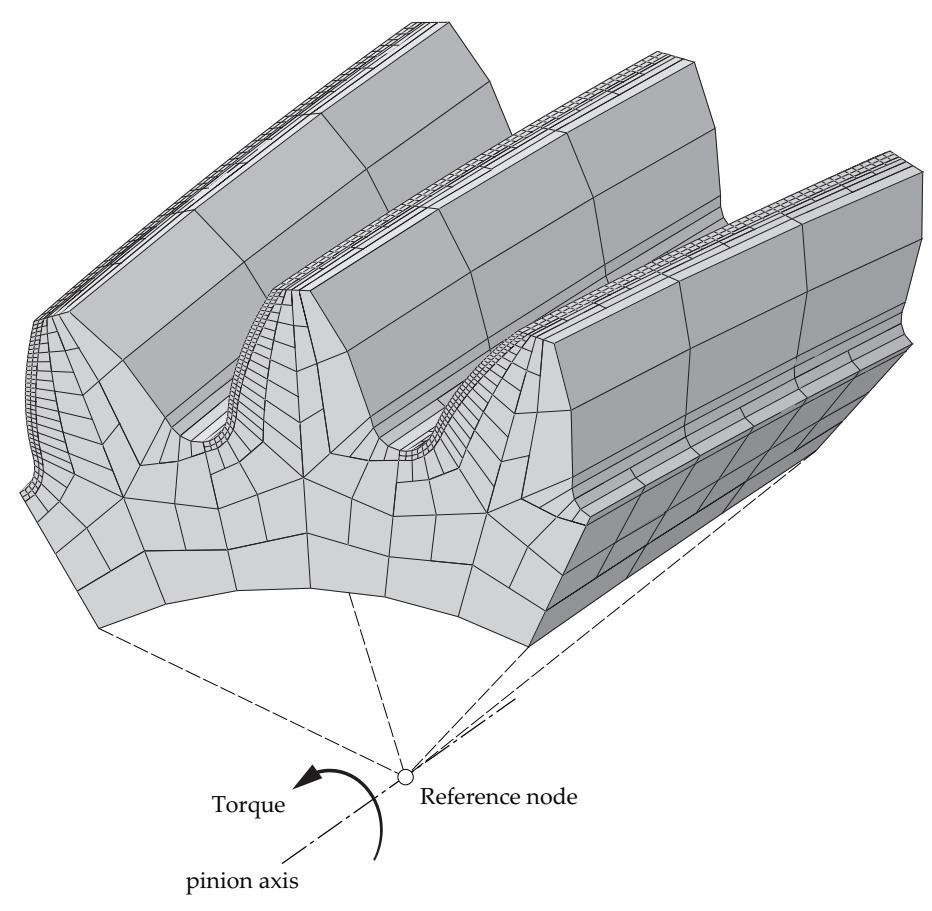

(a)

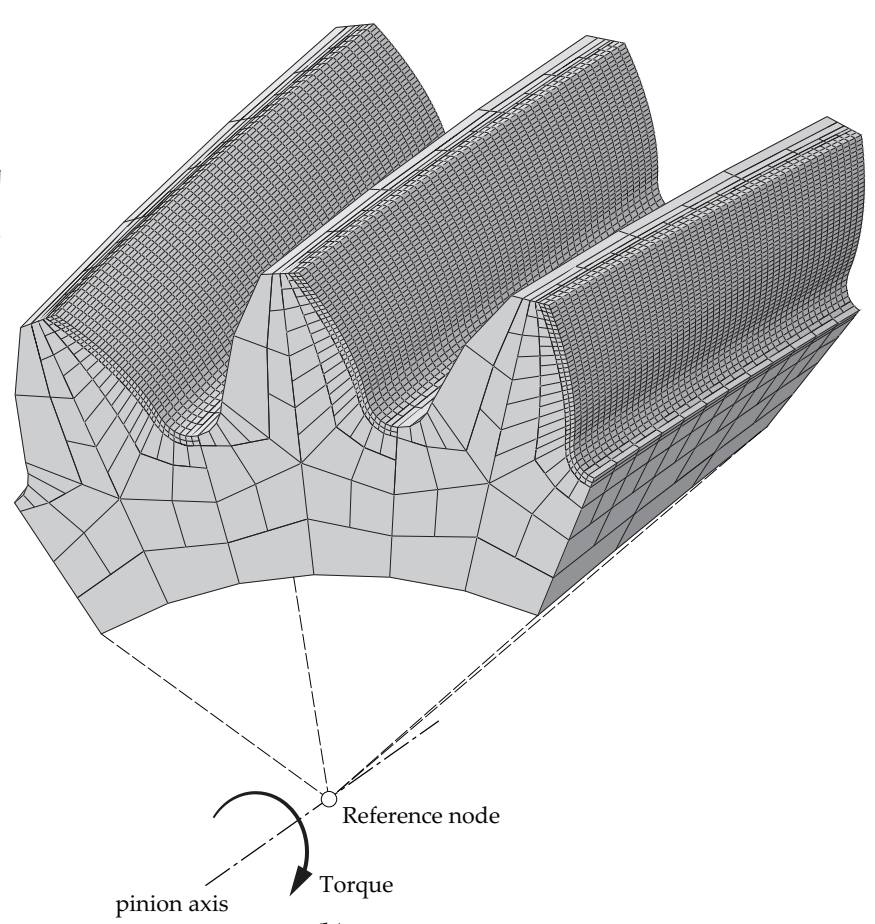

(b)

Figure 7: Finite element models of a right-hand helical pinion when it rotates: (a) counterclockwise, and (b) clockwise.

\subsection{Validation of the proposed model with Hertz theory}

An approach based on Hertz theory was implemented in [14] for stress analysis of gear drives with double crowned gear tooth surfaces and a localized bearing contact. The same numerical example is considered here and summarized in Table 1 for comparison of the obtained results with those provided by the proposed finite element model.

A finite element model of five pairs of teeth is considered (see Fig. 8). The use of five pairs of teeth allows the boundary constraints provided by the rigid surfaces of the pinion and the gear models to be far enough from the contact areas. The number of elements at contact and fillet regions are (see subsection 2.3) $n_{l}=64, n_{p}=64$ and $n_{f}=16$. A total of 118240 elements with 174644 nodes are considered. A contact interaction based on master gear tooth surfaces with slave pinion gear tooth surfaces is considered [13]. Several analyses considering different values of the thickness coefficient $c_{t}$ of the two contacting layers (see Fig. 8) have been considered (0.05, $0.075,0.1,0.125,0.15,0.175$, and 0.2). Initially, the analyses are carried out at just the mean contact position where the Hertz results are provided. Figure 9 shows the results corresponding to the maximum contact pressure $p_{o}$, contact area $A_{c}$, and maximum Tresca stress (with an average threshold [13] of 75\%) $\sigma_{T, o}$, for the different analyses. Different strategies to consider reduced integration elements are illustrated as well: (1) with no reduced-integration elements, (2) with reduced-integration elements at layers $1,2,3,7$, and 8 , and (3) with reduced-integration elements at layers $1,2,3,4,7$, and 8 .

A minimum for the maximum contact pressure $p_{o}$ is observed for a given thickness coefficient at each applied strategy. The relative error $\varepsilon$ respect to Hertz results is illustrated as well for the thickness coefficient where contact pressure is minimum. Although it is shown that the relative 
Table 1: Data of a spur gear drive and Hertz results.

\begin{tabular}{lr}
\hline Number of teeth of the pinion, $N_{1}$ & 21 \\
Number of teeth of the gear, $N_{2}$ & 50 \\
Module, $m[\mathrm{~mm}]$ & 4.0 \\
Pressure angle, $\alpha$ degrees] & 25.0 \\
Face width, $F[\mathrm{~mm}]$ & 40.0 \\
Parabola coefficient for profile crowning,$a_{p}\left[\mathrm{~mm}^{-1}\right]$ & 0.00025 \\
Parabola vertex location for profile crowning, $u_{o}[\mathrm{~mm}]$ & 0.0 \\
Parabola coefficient for longitudinal crowning, design C $(\mathrm{see}[14]), a_{l}\left[\mathrm{~mm}^{-1}\right]$ & 0.0004 \\
Parabola vertex location for longitudinal crowning,$l_{o}[\mathrm{~mm}]$ & 0.0 \\
Applied torque, $T[\mathrm{Nm}]$ & 200.0 \\
Young's module, $E[\mathrm{MPa}]$ & 206800.0 \\
Poisson's ratio, $\nu$ & 0.29 \\
Maximum contact pressure at the mean contact position, $p_{o}[\mathrm{MPa}]$ & 1241.2 \\
Maximum effective Tresca stress at the mean contact position, $\sigma_{T, o}[\mathrm{MPa}]$ & 761.5 \\
Semi-length of the major axis of the contact ellipse, $a[\mathrm{~mm}]$ & 7.514 \\
Semi-length of the minor axis of the contact ellipse, $b[\mathrm{~mm}]$ & 0.268 \\
Contact area, $A_{c}\left[\mathrm{~mm}^{2}\right]$ & 6.322 \\
\hline
\end{tabular}

error for the maximum contact pressure in strategy (2) is lower than in strategy (1), this fact is just circumstantial. Actually, contact areas $A_{c}$ are much further from Hertz results for strategies (2) and (3) than for strategy (1). The evaluation is that reduced-integration elements are stiffer, so the contact stresses and contact pressures are much lower as larger is the number of reduced-integration elements in the model.

On the other hand, it is observed a good approximation to the maximum contact pressure in any of the considered strategies, although the values of contact areas and maximum Tresca stresses are far from the results provided by Hertz theory. In fact, a larger number of elements underneath the contacting surfaces and along a depth equal to the length of the semi-minor axis $b$ would be required to improve these results, as it is illustrated in [14]. The problem of the finite element model presented in [14] is that it is not operative as the mesh would need to be adjusted for each contact position as well as it would have a very high computational cost.

\subsection{Comparison of the proposed model and the previous model}

A model as the one shown in Fig. 1 is considered here for comparison with the proposed model. Details and features of this model can be found in [12].

Figure 10(a) shows the evolution of the maximum contact pressure $p_{o}$ along two cycles of meshing distributed along twenty one contact positions. The contact positions are obtained from application of a Tooth Contact Analysis (TCA) algorithm [15] wherein three pairs of contacting teeth are taken into account. Results of TCA algorithm are illustrated in Figures 10(c) and 10(d). Two configurations of the proposed finite element model (as the one shown in Fig. 8) are considered in the results shown in Fig. 10(a), with $n_{p}=64$ and $n_{p}=48$, respectively, keeping $n_{l}=64$ and $n_{f}=16$ for both configurations. Completed integration elements are considered here. The computation time for both analyses along the two cycles of meshing are represented as well. Results 


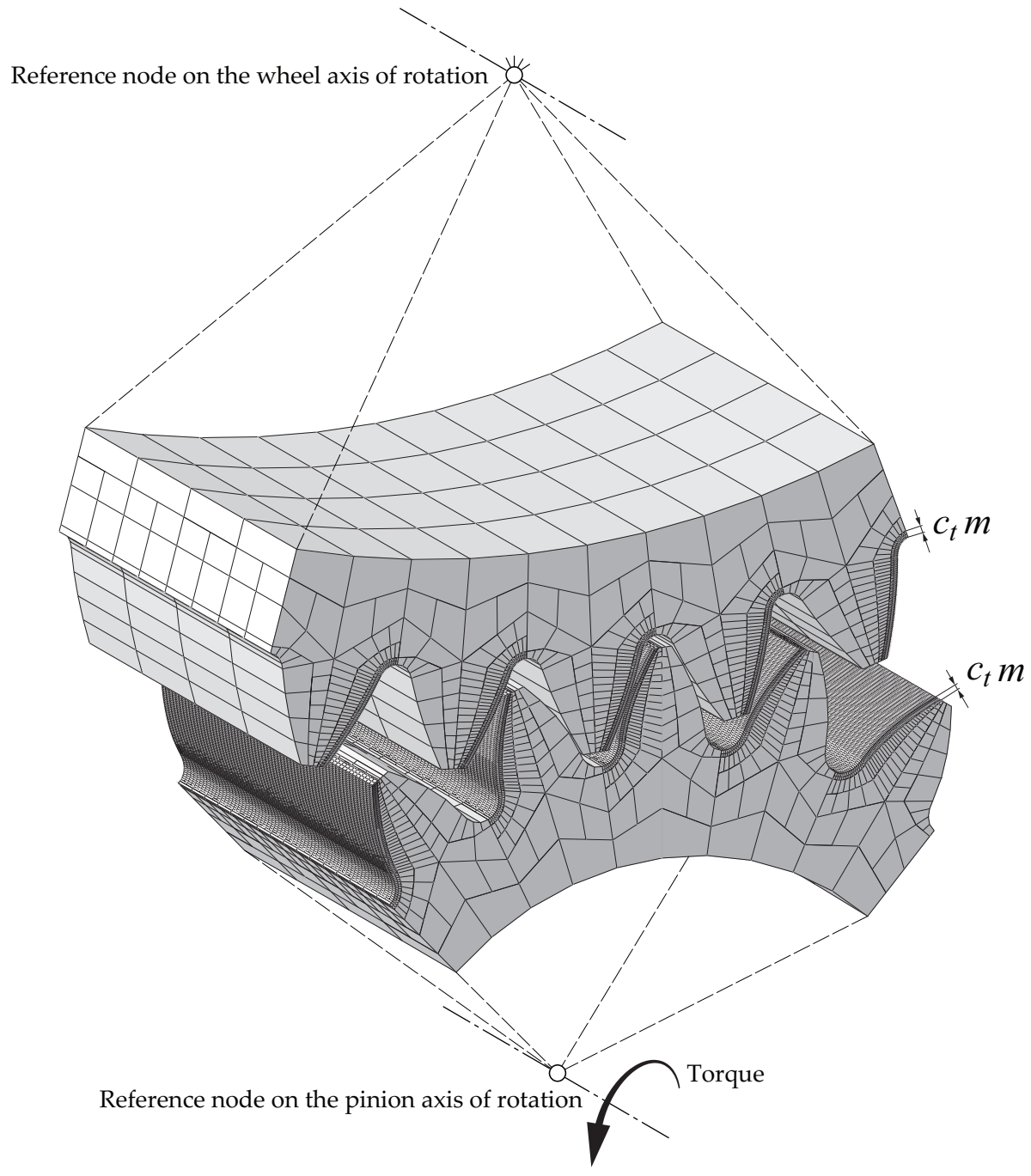

Figure 8: Finite element model for comparison of stresses with Hertz results and illustration of coefficient $c_{t}$.

provided by the model with $n_{p}=48$ show a good agreement with those obtained using the model with $n_{p}=64$. A reduction in computational time is observed as well.

Figure 10(b) shows the evolution of $p_{o}$ obtained from the proposed model with $n_{l}=64, n_{p}=48$, and $n_{f}=16$ and from the previous model (as the one shown in Fig. 1) with the same number of nodes in the contacting surfaces. The depth of the contacting layer of the previous model has been modified and now is constant and equal to the depth of the contacting layer of elements of the proposed model. The proposed model has 94360 elements with 139894 nodes. The previous model (for the same number of nodes on the contacting surfaces as the proposed model) has 286720 elements with 345932 nodes. Results of the evolution of $p_{o}$ along the two cycles of meshing show a good agreement between both models. The maximum relative error is $\varepsilon=4.3 \%$. However, the computational time is much lower for the proposed model than that for the previous model. The illustrated computational time is for two cycles of meshing and twenty one contact positions.

Finite element models with reduced integration elements (considering strategies (2) and (3) as it is illustrated in Fig. 9) were also investigated considering two cycles of meshing. They give similar 

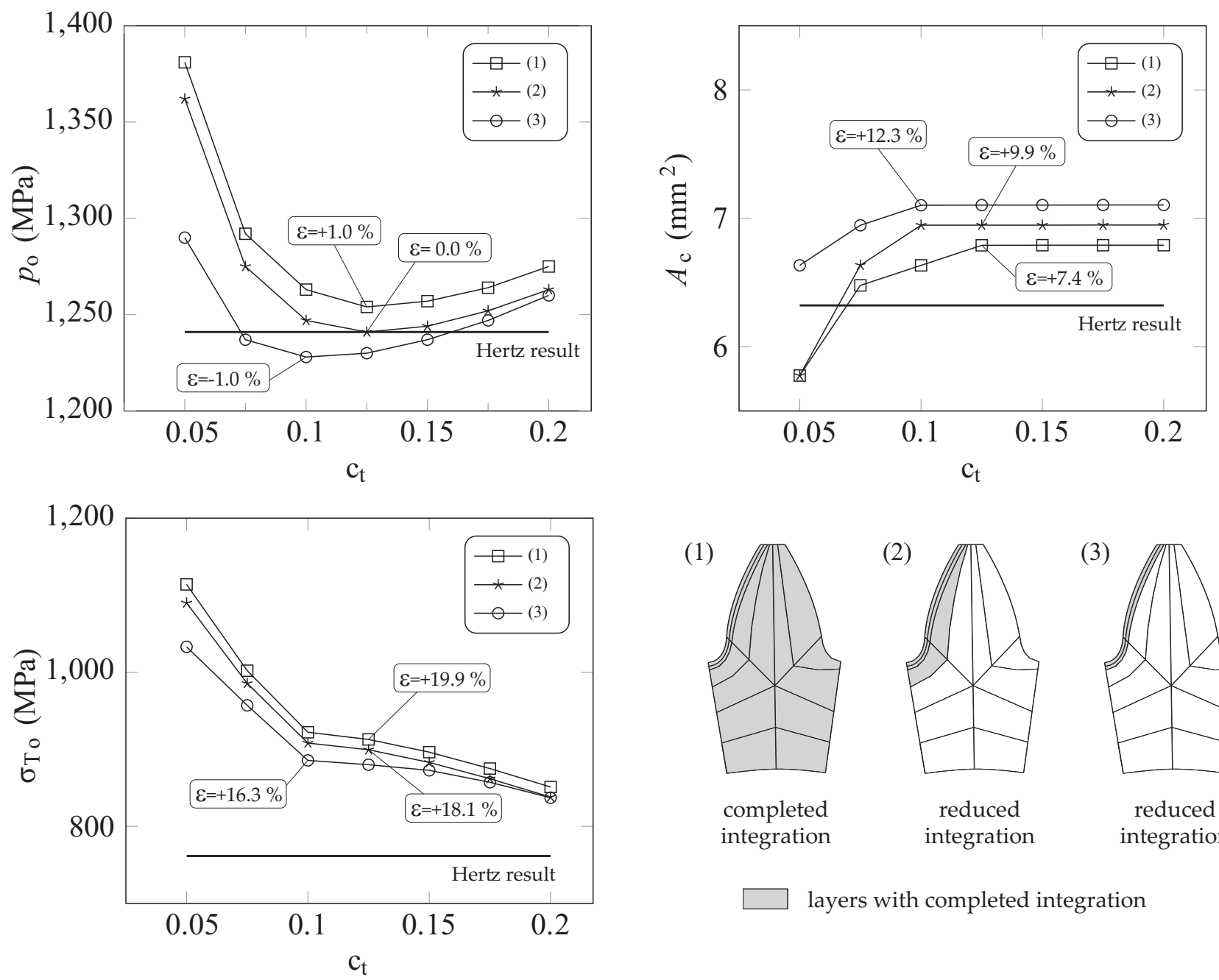

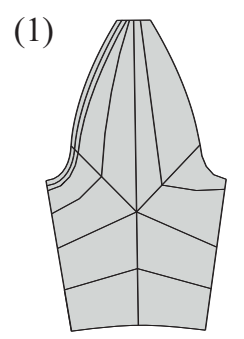

completed integration
(2)

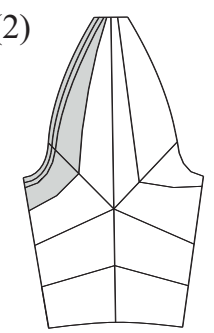

reduced integration
(3)

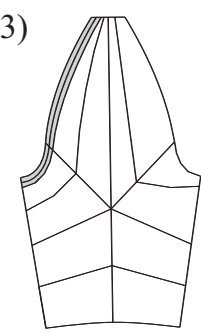

reduced integration

layers with completed integration

Figure 9: Comparison of the results obtained by the finite element analysis with those obtained by application of Hertz theory at the mean contact position and considering different layer thickness coefficients $c_{t}$ and different strategies for arrangement of reduced-integration elements: maximum contact pressure $p_{o}$ (up-left), contact area $A_{c}$ (up-rigth), and maximum Tresca stress (down-left).

results regarding $p_{o}$ evolution to the model with complete integration elements, but reduction of time is not significant. The computational time is 21.4 minutes for strategy (2) and 20.7 minutes for strategy (3) versus the 21.5 minutes of strategy (1) with complete integration elements.

\subsection{Validation of the proposed model regarding bending stresses}

The validation of the proposed model in regard to bending stresses is executed in two stages. In the first stage, the previous model similar to the one shown in Fig. 1 is considered. Nine analyses are executed at the mean contact position considering different mesh sizes at the fillet region in order to observe if results converge. In the second stage, the proposed and the previous models are compared considering two cycles of meshing in order to test the proposed model in regard to bending stresses. 

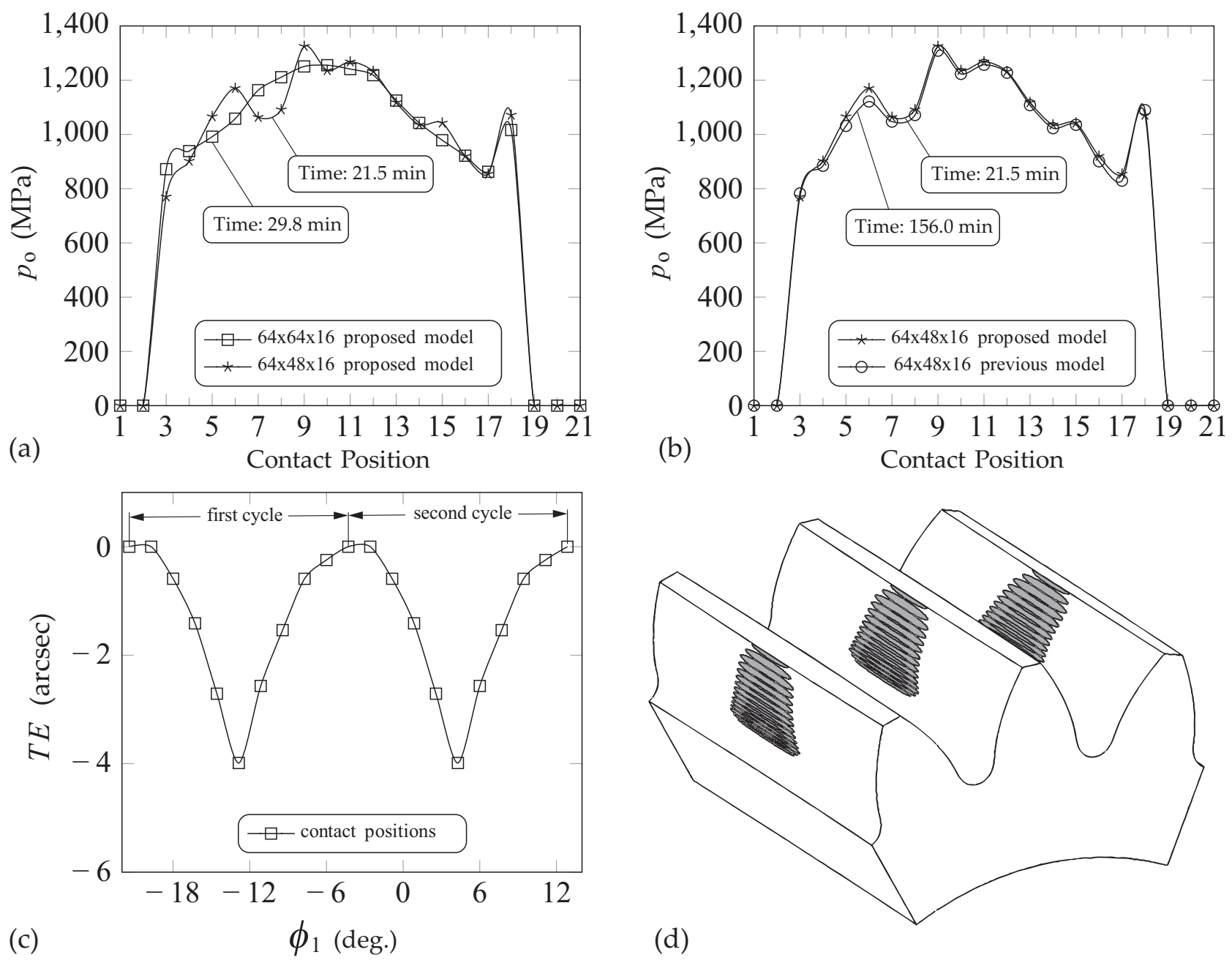

Figure 10: For comparison of proposed model and previous model: evolution of maximum contact pressure $p_{o}$ along two cycles of meshing at the middle pair of contacting teeth for (a) the proposed model with different configurations, and (b), the proposed model and the previous model; (c) function of transmission errors (TE) along pinion rotation $\phi_{1}$, and (d) formation of the bearing contact obtained from TCA algorithm.

The maximum principal stress at the fillet region of the middle tooth, $\sigma_{1 o}$, is investigated. Finite element models similar to the one shown in Fig. 1 with $n_{l}=64$ and $n_{p}=48$ are built. At each model, $n_{f}$ takes one of these values $n_{f}=\{2,4,6,8,10,12,14,16,18\}$ to build the nine finite element models. Figure 11(a) shows the values of $\sigma_{1 o}$ at the different models. Convergency of the results is observed. Relative errors for $n_{f}=8$ and $n_{f}=16$ are illustrated considering as a reference the value obtained for $n_{f}=18$.

In the second stage, the previous model with $n_{f}=16$ is considered as a reference to test the proposed model. Two cycles of meshing are considered. Figure 11(b) shows the evolution of $\sigma_{1 o}$ along the two cycles of meshing (see Fig. 10(c)). Here, the proposed model is built with complete integration elements. Two configurations are considered, with $n_{f}=\{8,16\}$. The maximum relative error occurs at contact position 12. A more rigid behaviour of the proposed model explains the low values of bending stresses respect to those that are obtained using the previous model. Similar 
results are observed for the wheel in Fig. 11(c).

Figure 11(d) shows the differences in the field of maximum principal stresses at the fillet of the middle tooth for the previous and the proposed models. The area of transition that was designed for the fillets of the proposed model (see Fig. 6) alters the field of stresses, although the maximum value has a relative error about $12.0 \%$.
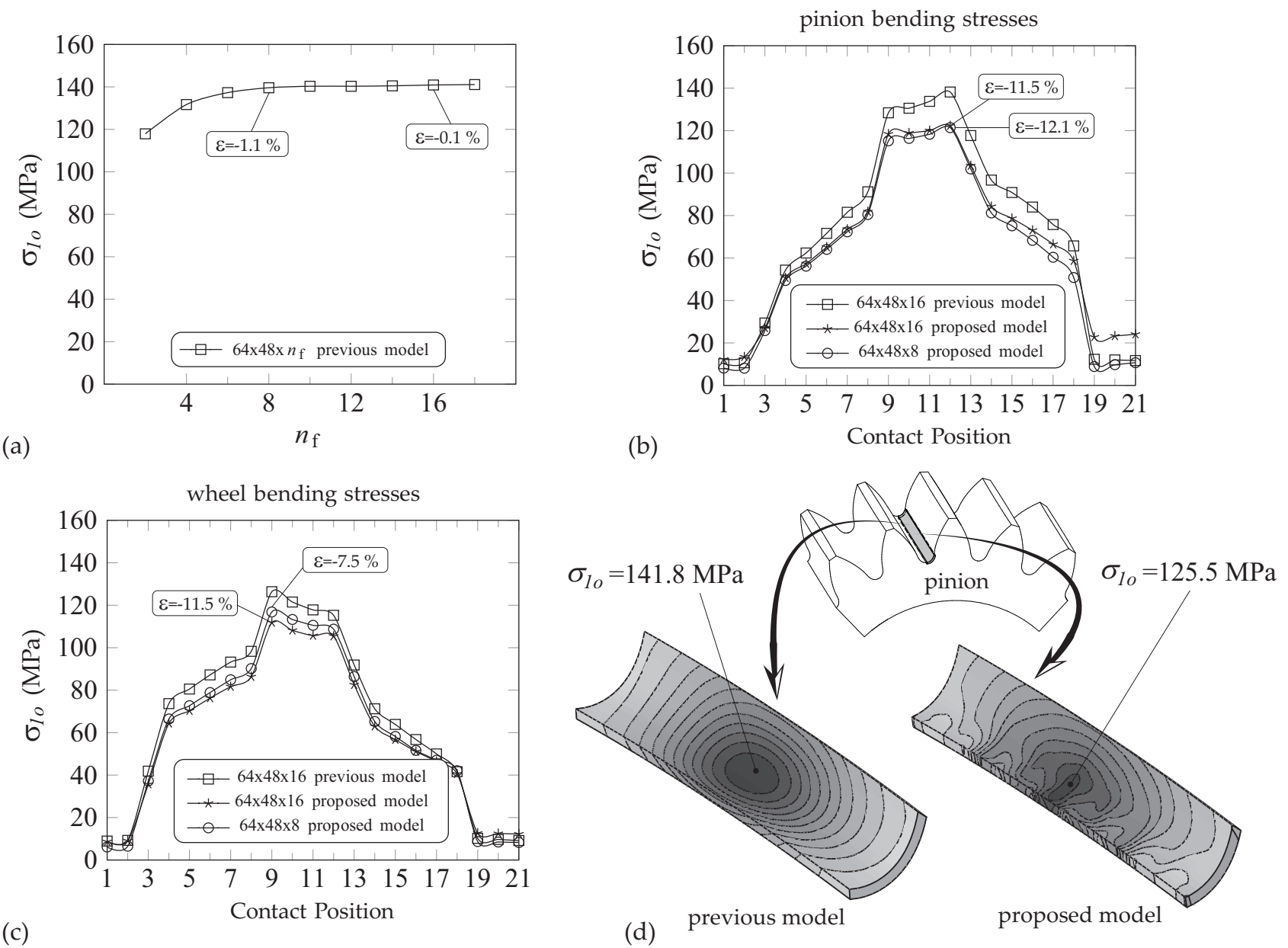

(c)

Figure 11: For validation of the proposed model regarding bending stresses: (a) maximum principal stress $\sigma_{1 o}$ at the fillet of the middle tooth of the pinion for different configurations of the previous model (contact at mean contact position), (b) comparison of the evolution of $\sigma_{1 o}$ at the pinion along the cycle of meshing, (c) comparison of the evolution of $\sigma_{1 o}$ at the wheel along the cycle of meshing, and (d) comparison of the field of $\sigma_{1 o}$ at the mean contact position.

\section{Conclusions}

The performed research allows the following conclusions to be drawn:

(1) A new finite element model based on the application of multi-point constraints and reducedintegrated elements has been proposed. It allows for a considerable reduction of the computational cost in the analysis of the whole cycle of meshing, mainly due to the reduction of 
the number of nodes and elements provided by the multi-point constraints rather than by the use of reduced-integration elements.

(2) The proposed finite element model can provide accurate results of maximum contact pressure as previous finite element models do, but with a lower computational cost. However, maximum effective Tresca stress (and for the same reason, maximum effective Von Mises stress) is not captured neither by the proposed model nor the existing model. More elements underneath the contacting surfaces would be required as it is illustrated in [14].

(3) A more rigid behaviour of the teeth in the proposed model provides lower values of bending stresses along the whole cycle of meshing, with relative errors about $10 \%$, respect to the results provided by the previous model.

\section{Acknowledgments}

The authors express their deep gratitude to the Spanish Ministry of Education, Culture, and Sport, for the financial support received through the scholarship Ref. PRX16/00416 from the Program of Mobility Stays for Senior Professors and Researchers at Foreign Centers for High Level Education and Research, in the frame of the Spanish State Program for Scientific and Technical Research and Innovation 2013-2016.

\section{References}

[1] L. Wilcox, W. Coleman, Application of Finite Elements to the Analysis of Gear Tooth Stresses, Journal of Engineering for Industry 95 (4) (1973) 1139.

[2] R. F. Handschuh, F. L. Litvin, A method for determining spiral-bevel gear tooth geometry for finite element analysis, NASA Technical Paper 3096, AVSCOM Technical Report 91-C-020 (1991) 1-20.

[3] J. J. Coy, C. H. Chao, A method of selecting grid size to account for Hertz deformation in finite element analysis of spur gears, Nasa Technical Memorandum 86263, AVRADCOM Technical Report 81-C-14 (1981) 1-26.

[4] G. D. Bibel, K. Tiku, A. Kumar, R. F. Handschuh, Comparison of Gap Elements and Contact Algorithm for 3D Contact Analysis of Spiral Bevel Gears, NASA Technical Memorandum 106643, Technical Report ARL-TR-478.

[5] J. H. Argyris, A. Fuentes, F. L. Litvin, Computerized Integrated Approach for Design and Stress Analysis of Spiral Bevel Gears, Computer Methods in Applied Mechanics and Engineering 191 (11-12) (2002) 1057-1095.

[6] I. Gonzalez-Perez, V. Roda-Casanova, A. Fuentes, F. T. Sanchez-Marin, J. L. Iserte, A finite element model for consideration of the torsional effect on the bearing contact of gear drives, Journal of Mechanical Design, Transactions of the ASME 134 (071007) (2012) 1-8.

[7] A. Fuentes, R. Ruiz-Orzaez, I. Gonzalez-Perez, Compensation of errors of alignment caused by shaft deflections in spiral bevel gear drives, Vol. 34, 2016.

[8] S. M. Vijayakar, A combined surface integral and finite element solution for a threedimensional contact problem, International Journal for Numerical Methods in Engineering 31 (1991) 525-545.

[9] M. Kolivand, A. Kahraman, A load distribution model for hypoid gears using ease-off topography and shell theory, Mechanism and Machine Theory 44 (10) (2009) 1848-1865.

[10] S. M. Vijayakar, Finite element methods for quasi-prismatic bodies with application to gears, Ph.D. thesis, The Ohio State University (1987).

[11] K. Mao, Gear tooth contact analysis and its application in the reduction of fatigue wear, Wear 262 (11-12) (2007) 1281-1288.

[12] F. L. Litvin, A. Fuentes, Gear Geometry and Applied Theory, Cambridge University Press, 2004.

[13] Dassault Systemes, Inc., 2015, ABAQUS/Standard Analysis User's Guide, 175 Wyman Street, Waltham, Massachusetts, United States.

[14] I. Gonzalez-Perez, J. L. Iserte, A. Fuentes, Implementation of Hertz theory and validation of a finite element model for stress analysis of gear drives with localized bearing contact, Mechanism and Machine Theory 46 (6) (2011) 765-783. 
[15] A. Fuentes, R. Ruiz-Orzaez, I. Gonzalez-Perez, Computerized design, simulation of meshing, and finite element analysis of two types of geometry of curvilinear cylindrical gears,Computer Methods in Applied Mechanics and Engineering 272 (2014), 321-339. 